\title{
Características de manejo y conducta en caballos estabulados en el sur de Chile: Estudio preliminar
}

\author{
Husbandry and behaviour characteristics in stabled horses in \\ the south of Chile: A preliminary study \\ C Márquez ${ }^{\mathrm{a}}$, A Escobar ${ }^{\mathrm{ab}}$, TA Tadich ${ }^{\text {ab* }}$ \\ ${ }^{a}$ Instituto de Ciencia Animal, Facultad de Ciencias Veterinarias, Universidad Austral de Chile, Valdivia, Chile. \\ bPrograma Bienestar Animal, Facultad de Ciencias Veterinarias, Universidad Austral de Chile, Valdivia, Chile.
}

\begin{abstract}
SUMMARY
The use of stables for horses has become a routine practice, especially for those used in equestrian sports, affecting some behaviours due to the changes in their natural environment which can have a detrimental effect on their welfare. In Chile the conditions under which horses are kept in stables in relation to welfare have not been described. This is why the main objective of this study was to describe the housing systems for horses used in the south of Chile and determine if they have the conditions required to maintain an adequate welfare status. Fourteen stud farms, including 283 horses of different breeds located in Valdivia, Ranco, Osorno and Chiloé provinces were visited. Direct and indirect methods were used for the evaluation; these included aspects of health, behaviour, handling of the horses and training of the personnel. According to our results most horses had a good body condition score $(88 \%), 2.5 \%$ presented lesions and only $0.7 \%$ were lame. All stud farms had deworming schedules and the majority a vaccination programme. The main problems were related with the presence of behavioural disorders (18.4\%), low number of food rations per day, size of the stables and lack of training of the personnel, especially in relation to animal welfare concepts. All stud farms had a high level of concern about the care of their horses. However, the lack of knowledge in some aspects related to horse management need to be addressed through training in order to improve horses welfare.
\end{abstract}

Palabras clave: equinos, bienestar, manejo, estabulación.

Key words: equines, welfare, management practices, stables.

\section{INTRODUCCIÓN}

La estabulación del equino es una práctica que se ha ido masificando en los criaderos a través del tiempo; entre las razones de esto están la necesidad de ahorrar pasturas, eliminar la competencia durante la alimentación y facilitar a los dueños un mayor control sobre el valor nutritivo del alimento entregado y sobre la ingesta de agua (McGreevy 2004). Sin embargo, la estabulación puede tener un efecto negativo sobre el bienestar equino, principalmente debido a las modificaciones conductuales que sufren al reemplazar su ambiente natural por establos (McGreevy 2004). La conducta de forrajeo se ve altamente alterada en un sistema de estabulación. Los equinos en su ambiente natural pueden ocupar hasta 16 horas diarias forrajeando, mientras que en estabulación esta conducta se ve reducida a aproximadamente tres horas del día (Kiley-Worthington 1987), produciendo frustración alimenticia durante el encierro; la estabulación es también un factor de riesgo para la presentación de conductas anormales o no deseadas (Christie y col 2006, Tadich y Araya 2010).

\footnotetext{
Aceptado: 10.08.2010.

* Programa Bienestar Animal. Facultad de Ciencias Veterinarias, UACh, Casilla 567, Valdivia, Chile; tamaratadich@uach.cl
}

La evaluación del bienestar animal se basa principalmente en la recolección de dos tipos de información: los métodos indirectos, que evalúan lo adecuado de los insumos y las prácticas de manejo que el animal recibe e indican el riesgo de un problema de bienestar (Wood y col 1998), y los métodos directos, que usan parámetros basados en el animal proporcionando una medida del estado de bienestar de éste (Pritchard y col 2005). Dentro de los aspectos a evaluar se deben incluir aquellos relacionados con el comportamiento, mantención, alimentación y cuidado veterinario, elementos básicos para la mantención de equinos en condiciones adecuadas (Endenburg 1999).

Las condiciones en que se mantienen los equinos estabulados en relación a su bienestar no han sido descritas en Chile, por ello se planteó como objetivo describir los sistemas de estabulación en relación a la presencia de condiciones apropiadas para mantener un estado de bienestar adecuado de los equinos.

\section{MATERIAL Y MÉTODOS}

\section{ANIMALES}

Se utilizaron 283 equinos, en su mayoría destinados a deporte, de diversas razas, pertenecientes a 14 criaderos de la zona sur del país, ubicados en las Provincias de 
Valdivia y Ranco, Región de Los Ríos, y en las Provincias de Osorno y Chiloé, Región de Los Lagos.

\section{EVALUACIÓN DE BIENESTAR ANIMAL}

Se aplicó el protocolo de evaluación de bienestar animal aplicado por Márquez (2009), el cual contempla indicadores directos e indirectos.

Indicadores directos: fueron utilizados el estado de salud de los animales, incluyendo la condición corporal, según la tabla de condición corporal desarrollada por Henneke (1985) y modificada por Naour (2003), clasificando la condición corporal en buena, regular o mala; presencia de heridas y cicatrices, presencia de claudicaciones y lesiones de cascos observables a la inspección del animal, tales como: fisuras, desprendimiento de muralla e infecciones. Además, se observó la presencia de conductas anormales como lignofagia, aerofagia con o sin fijación, mal del oso, sacudir la cabeza, patear la pesebrera y caminar estereotipado, según las descripciones entregadas por Tadich y Araya (2010). Estos indicadores se evaluaron por inspección directa de los equinos dentro de sus pesebreras. En el caso de los indicadores conductuales, estos se registraron mediante el método de escaneo focal, por el tiempo que duró la visita al criadero, en promedio 4 horas, y se contrastó con lo indicado por los respectivos cuidadores por medio de encuesta.

Indicadores indirectos: fueron utilizados los programas de manejo sanitario (vacunas y desparasitaciones), infraestructura de los establos (tamaño, iluminación y ventilación), manejo nutricional y alimenticio, tiempo de estabulación (horas por día), presencia y tipo de herraje, método de amansa utilizado (tradicional o racional) y capacitación del personal en manejo equino. Estos indicadores fueron evaluados mediante observación del establo y la aplicación de una encuesta en relación a manejo, confeccionada para los trabajadores de los criaderos que estaban en contacto permanente con los equinos estabulados.

\section{ANÁLISIS ESTADÍSTICO}

Se utilizó estadística descriptiva. Los resultados para las distintas observaciones se expresaron en base a números totales y porcentajes.

\section{RESULTADOS Y DISCUSIÓN}

De la evaluación de los 283 equinos incluidos en este estudio se evidenció que un $88 \%$ de ellos obtuvo una calificación de condición corporal buena; un 12\% una condición corporal regular y no se observó ningún animal con una calificación de condición corporal mala. Esto contrasta con lo reportado por Naour (2003) y Tadich y col (2008) quienes utilizaron la misma escala de evaluación de condición corporal en equinos de tiro liviano (carretoneros) encontrando $36 \%$ y $59 \%$ de los equinos en condición corporal buena y un $9 \%$ y $8 \%$ en condición corporal mala respectivamente. Las diferencias con los estudios mencionados podrían deberse a que los dueños de caballos carretoneros son en general personas de clase socioeconómica baja (Mac-Leod 1999), lo que dificulta la mantención de una buena condición corporal de los animales producto de la falta de recursos para comprar alimentos de buena calidad para satisfacer sus requerimientos nutricionales, los cuales probablemente se pueden solventar en los equinos de deporte involucrados en este estudio.

En cuanto a la presencia de heridas y cicatrices, se observó un bajo porcentaje de lesiones, siendo éstas de un $2,5 \%$ y un $8,8 \%$ respectivamente. Estos porcentajes son inferiores al $27 \%$ de heridas y $50 \%$ de cicatrices encontrados en equinos carretoneros por Tadich y col (2008). En dicho estudio la mayoría de las lesiones fueron provocadas por los aperos, los cuales, tal como señala Hovell (1998), causan lesiones producto de una pobre mantención o uso inapropiado; en cambio, los aperos utilizados en equinos de deporte presentan mejores características de calidad y ajuste de acuerdo a la función y necesidades particulares del equino (Del Campo 2004).

La prevalencia de problemas de comportamiento en este estudio fue de un $18,4 \%$, teniendo en cuenta que algunos equinos presentaban más de un problema conductual (cuadro 1). Este porcentaje es mayor al 10\% reportado en Chile para caballos chilenos por Muñoz y col (2009) y a resultados en otros países, donde se han encontrado prevalencias que van desde un 2,5\% en Italia (Vecchiotti y Galanti 1986) hasta un 15\% en Canadá (Luescher y col 1991). La alta prevalencia de problemas de comportamiento en el presente estudio se puede deber a que se consideró un mayor número de desórdenes conductuales

Cuadro 1. Distribución de los problemas de comportamiento observados en los equinos estabulados de los criaderos evaluados.

horses.

Distribution of the behavioural disorders observed in stabled

\begin{tabular}{lcc}
\hline Problema conductual & $\begin{array}{c}\text { Número } \\
\text { de equinos }\end{array}$ & $\begin{array}{c}\text { Porcentaje } \\
(\%)\end{array}$ \\
\hline Masticar madera (lignofagia) & 16 & 5,7 \\
Patear la pesebrera & 10 & 3,5 \\
Aerofagia con o sin fijación & 8 & 2,8 \\
Sacudir la cabeza & 6 & 2,1 \\
Mal del oso & 9 & 3,2 \\
Caminar estereotipado & 1 & 0,4 \\
Otro* & 2 & 0,7 \\
Ninguno & 233 & 82,3 \\
\hline
\end{tabular}

* Otro corresponde a morderse el labio continuamente y levantar la cola continuamente. 
que en los estudios mencionados, donde sólo tomaron en cuenta problemas como el mal del oso, aerofagia con fijación, caminar estereotipado y otros desórdenes como lignofagia. La mayor presencia de problemas de comportamiento podría atribuirse a la cantidad de horas de estabulación continua a la que eran sometidos los equinos en el presente estudio (cuadro 2), siendo éste un factor de riesgo para la presentación de conductas anormales (Christie y col 2006).

El mal del oso, la aerofagia con fijación y el caminar estereotipado se presentaron en un porcentaje levemente menor a los encontrados en otros estudios (cuadro 1). Waters y col (2002) reportaron un 4,6\% de equinos con el mal del oso, un 2,3\% con caminar estereotipado y $10,5 \%$ aerofagia con fijación. En tanto, Christie y col (2004) registraron prevalencia para estos comportamientos de un 4,8, 3,8 y 3,8\% respectivamente. Esto podría deberse a que, a pesar de su estabulación continua, todos los equinos tenían contacto entre ellos, siendo principalmente este contacto de tipo visual y en menor medida visual y táctil (cuadro 2), lo cual podría disminuir la presentación de problemas como el mal del oso (McGreevy y col 1995).

En cuanto al manejo sanitario, los equinos de los 14 criaderos evaluados $(100 \%)$ contaban con asistencia veterinaria periódica, siendo esto superior a lo señalado por Iturriaga (1998) en el país, quien encontró que sólo un $64,5 \%$ de los centros equinos en Chile contaba con asistencia veterinaria periódica. La totalidad de los criaderos realizaba tratamientos antiparasitarios a sus equinos en forma periódica, con fechas predeterminadas sin basarse

Cuadro 2. Características de la infraestructura y del manejo equino durante la estabulación.

Characteristics of the stables and husbandry practices used.

\begin{tabular}{llrr}
$\begin{array}{l}\text { Características de } \\
\text { la estabulación }\end{array}$ & & $\begin{array}{c}\text { Número } \\
\text { Porcentaje } \\
(\%)\end{array}$ \\
\hline $\begin{array}{llr}\text { Tamaño de } \\
\text { pesebreras (m) }\end{array}$ & $2 \times 5 \mathrm{v} 3$ & 2 & 14,2 \\
& $3 \times 3$ & 6 & 42,9 \\
Lugar de & Pesebreras & 6 & 42,9 \\
almacenamiento & Bodega y pesebrera & 3 & 7,1 \\
del alimento & Galpón o bodega & 10 & 71,4 \\
Frecuencia de & 2 veces/día & 8 & 57,1 \\
alimentación & 3 veces/día & 5 & 35,6 \\
& 4 veces/día & 1 & 7,1 \\
Tiempo de estabu- & $<2$ horas & 1 & 7,1 \\
lación & 2 - 4 horas & 5 & 35,7 \\
continua en el día & 4 - 8 horas & 6 & 42,9 \\
& $>8$ horas & 2 & 14,3 \\
Contacto entre & Visual & 11 & 78,6 \\
equinos estabulados & Visual y táctil & 3 & 21,4 \\
\hline
\end{tabular}

en exámenes coprológicos, en donde la mayor parte de éstos la realizaban cada tres meses $(78,6 \%)$. Esta forma de manejo puede afectar el bienestar animal de los equinos debido a que produce resistencia de los parásitos hacia los fármacos antihelmínticos, como se ha reportado en el caso de los ciatostomas a los bencimidazoles (Herd y Coles 1995), lo cual se ha reportado en equinos del sur de Chile (von Witzendorff y col 2003).

La mayoría de los criaderos utilizaban vacunas para prevenir algunas enfermedades infecciosas (influenza equina, rinoneumonitis y gurma); sólo se encontró un $14,3 \%$ de criaderos que no aplicaban este método preventivo. Esto se debe a que los dueños de los criaderos en cuestión no han tenido problemas de esta naturaleza, por lo cual no consideran necesario este manejo; aun así, este porcentaje es menor al 22,6\% reportado hace ya una década por Iturriaga (1998).

En cuanto a la infraestructura, el 100\% de los establos contaba con ventilación, dada principalmente por aperturas en el techo y por ventanas, tal como recomienda Ensminger (1973). Todos los criaderos tenían en sus establos luz natural y luz artificial lo que, según Retamales (1989), es importante para evitar la sensación de encierro en los animales. Las características que presentaban las pesebreras diferían entre los criaderos principalmente en tamaño y en algunos casos en el uso que se les daba, en relación al almacenamiento de alimento (cuadro 2).

Más del 50\% de los criaderos evaluados presentaba pesebreras con dimensiones iguales o menores a $3 \times 3$ metros, siendo que esas son las dimensiones mínimas recomendadas para equinos de alzadas menores a $1,42 \mathrm{~m}$ (Webster y col 1987). A pesar de no haberse realizado una clasificación de los equinos según su alzada, se estima que la mayoría de los animales involucrados en el estudio era de una alzada mayor. La utilización de pesebreras de tamaños menores a los recomendados limita el movimiento y actividad de los equinos al interior de éstas, provocando así distrés en el animal, impidiendo que exhiban un repertorio conductual adecuado y repercutiendo en la posible aparición de conductas estereotipadas (Broom y Kennedy 1993).

La utilización de las pesebreras como lugar de almacenamiento de alimento (cuadro 2) no es aconsejable, ya que éste puede atraer roedores que transmiten enfermedades, además el heno en mal estado favorece la presentación de problemas respiratorios como la obstrucción recurrente de las vías aéreas (Clarke 1987, Morán y col 2006).

En cuanto a la alimentación y estabulación, en el cuadro 2 se observan algunos aspectos importantes que se pueden relacionar con el bienestar de estos equinos. En la mayoría $(57,1 \%)$ de los criaderos evaluados la frecuencia de alimentación más usada era de dos veces/ día. Esta frecuencia resulta insuficiente y provoca una reducción importante de la conducta de forrajeo (KileyWorthington 1987). Un bajo número de raciones repercute negativamente en el sistema digestivo ya que facilita la 
presentación de úlceras gástricas; éstas como consecuencia de una disminución del pH gástrico, provocada por la secreción continua de ácido clorhídrico que realizan los equinos (Andrews y col 2005) y que, en estos casos, no es amortiguado correctamente por la secreción salival, debido a los largos periodos de ayuno a los que son sometidos. Además un reducido tiempo dedicado al forrajeo podría causar frustración alimenticia causando cambios conductuales. Una frecuencia de alimentación recomendable es de cuatro veces al día, ya que un aumento en la frecuencia previene la aparición de conductas anormales (Ninomiya y col 2004) y al mismo tiempo disminuyen la probabilidad de aparición de úlceras gástricas, ya que la entrega, cada seis horas, de forrajes ricos en fibras ayuda a regular el pH estomacal (Andrews y col 2005) a través de una adecuada producción de saliva.

En cuanto al tiempo de estabulación, sobre el $50 \%$ de los criaderos mantenía a sus animales estabulados por más de cuatro horas continuas (cuadro 2), factor de riesgo importante en la presentación de problemas de comportamiento (Mills y col 2005).

La capacitación del personal en diferentes ámbitos relacionados a los equinos se muestra en el cuadro 3. En el caso de los herrajes la mayoría de los criaderos contaba con una persona capacitada para realizar este manejo. La práctica del herraje es una actividad fundamental que requiere conocimientos adecuados para realizarlo sin provocar daños en el pie del equino y es indispensable que el herrador esté formado académicamente (Funtanillas 2004).

El 0,7\% de los equinos presentaba algún tipo de claudicación y un 9,9\% problemas a nivel de cascos, porcentajes inferiores a lo reportado por Mac-Leod (1999) y Naour (2003) en equinos carretoneros, quienes encontraron $13,5 \%$ y $10 \%$ de prevalencia de claudicaciones y $90,4 \%$ y $93 \%$ de problemas en los cascos, respectivamente. Los bajos porcentajes encontrados podrían ser producto de que la mayoría de los criaderos $(85,7 \%)$ contaba con gente capacitada en herraje y el $100 \%$ de los criaderos evaluados efectuaban despalme y cambio de herraje con intervalos de cuatro a seis semanas, coincidiendo con lo recomendado por la World Society for the Protection of Animals (WSPA 2003).
La amansa de los caballos la efectuaba mayoritariamente personal sin capacitación en el tema (60\%), lo que podría afectar el bienestar de los equinos, ya que en general éstos utilizan la amansa tradicional (80\%), la cual se basa principalmente en el uso de castigos (Porte 1979), mientras que sólo un $20 \%$ practicaba amansa racional (cuadro 3), basada en el comportamiento y refuerzo positivo del caballo (Muñoz 2004).

La capacitación del personal en el manejo equino y bienestar animal demostró ser un aspecto que todavía no es considerado como elemento importante. Mejorar los porcentajes de capacitación, especialmente en el tema de bienestar animal, podría mejorar las condiciones de los equinos. Altamirano (2004) demostró cómo la capacitación de trabajadores con respecto a temas de manejo animal resultó en mejoras en los indicadores de bienestar animal en plantas faenadoras.

En general, la buena condición corporal en que se encontraban los equinos denota que existe preocupación por el cuidado de ellos. Sin embargo, la presencia de problemas de comportamiento es un aspecto preocupante. Una manera de enfrentar este aspecto es dar a conocer los factores de riesgo y consecuencias de estos desórdenes conductuales sobre la salud de los equinos. Otros problemas que se encontraron se relacionan con desconocimiento de las necesidades referentes a espacio, conducta normal y frecuencia de alimentación requerida por los equinos, aspectos que pueden ser solucionados a través de capacitación del personal que maneja de forma directa a los equinos en los criaderos.

\section{RESUMEN}

La estabulación de los equinos es una forma de manejo cotidiana, principalmente en aquellos destinados a deporte, provocando modificaciones conductuales debido al cambio desde un ambiente natural a uno artificial, pudiendo afectar su bienestar. En Chile, las condiciones en que se mantienen los equinos estabulados en relación a su bienestar no han sido descritas. El objetivo de este estudio fue describir algunas de las condiciones de estabulación de equinos y determinar si éstas son las requeridas para mantener un estado de bienestar adecuado. Se evaluaron 283 equinos, de distintas razas, pertenecientes a 14 criaderos del sur de Chile (Provincias de Valdivia, Ranco, Osorno y Chiloé). Se utilizaron métodos directos e indirectos; éstos consideraron aspectos de

Cuadro 3. Capacitación del personal en distintos aspectos asociados al manejo equino.

Training of handlers in different aspects associated with equine management.

\begin{tabular}{|c|c|c|c|c|c|c|c|c|}
\hline \multirow{2}{*}{ Capacitación } & \multicolumn{2}{|c|}{ Herraje } & \multicolumn{2}{|c|}{ Amansa* } & \multicolumn{2}{|c|}{ Manejo general } & \multicolumn{2}{|c|}{ Bienestar animal } \\
\hline & $\mathrm{N}^{\mathrm{o}}$ & $\%$ & $\mathrm{~N}^{\mathrm{o}}$ & $\%$ & $\mathrm{~N}^{\mathrm{o}}$ & $\%$ & $\mathrm{~N}^{\mathrm{o}}$ & $\%$ \\
\hline Sí & 12 & 85,7 & 4 & 40 & 7 & 50 & 1 & 7,1 \\
\hline No & 2 & 14,2 & 6 & 60 & 7 & 50 & 13 & 92,9 \\
\hline
\end{tabular}

* Sólo 10 de los criaderos evaluados realizaban la amansa. 
salud, comportamiento, prácticas de manejo y capacitación del personal. El 88\% de los equinos presentó buena condición corporal, mientras que pocos presentaron heridas $(2,5 \%)$ y claudicaciones $(0,7 \%)$. Todos los criaderos se preocupaban de realizar manejo antiparasitario y la mayoría de vacunar contra enfermedades infecciosas. Las principales falencias fueron la presencia de problemas conductuales $(18,4 \%)$; la baja frecuencia de la alimentación de los equinos, tamaño de las pesebreras y la capacitación del personal en el manejo equino, particularmente la capacitación en relación al bienestar animal. En todos los criaderos existe preocupación por el cuidado de sus animales, sin embargo, la falta de conocimiento en relación a los problemas presentados es una situación que debería ser solucionada. La capacitación del personal encargado del manejo equino, en cuanto a comportamiento normal, sería un aporte para mejorar el bienestar de estos animales.

\section{REFERENCIAS}

Altamirano A. 2004. Evaluación del bienestar animal mediante la observación de tres indicadores en una planta faenadora de carnes de bovino. Memoria de título, Escuela de Medicina Veterinaria, Universidad Austral de Chile, Valdivia, Chile.

Andrews FM, BR Buchanan, SB Elliot, NA Clariday, LH Edwards. 2005. Gastric ulcers in horses. J Anim Sci 83, Suppl E18-E21.

Broom DM, MJ Kennedy. 1993. Stereotypies in horses: their relevance to welfare and causation. Equine Vet Educ 5, 151-153.

Christie JL, CJ Hewson, CB Riley, MA McNiven, IR Dohoo, LA Bate. 2004. Demographics, management and welfare of nonracing horses in Prince Edward Island. Can Vet J 45, 1004-1011.

Christie JL, CJ Hewson, CB Riley, MA McNiven, IR Dohoo, LA Bate. 2006. Management factors affecting stereotypies and body condition score in nonracing horses in Prince Edward Island. Can Vet J 47, 136-143.

Clarke AF. 1987. Air hygiene and equine respiratory disease. In Pract 9 , 196-204.

Del Campo CH. 2004. A la huella: caballos, aperos y cabalgatas. Imprenta América Ltda., Valdivia, Chile.

Endenburg N. 1999. Perceptions and attitudes towards horses in European societies. Equine Vet J, Suppl 28, 38-41.

Ensminger M. 1973. Producción equina. 6a ed. El Ateneo, Buenos Aires, Argentina.

Funtanillas H. 2004. Elementos de podología equina y herrado correctivo. Editorial Hemisferio Sur, Buenos Aires, Argentina.

Henneke DR. 1985. A condition score system for horses. Equine Pract 7, 13-15.

Herd RP, G Coles. 1995. Slowing the spread of anthelmintic-resistant nematodes of horses in the United Kingdom. Vet Rec 136, 481-485.

Hovell G. 1998. Welfare considerations when attaching animals to vehicles. Appl Anim Behav Sci 59, 11-17.

Iturriaga L. 1998. Estudio descriptivo de 31 centros reproductivos equinos en la Décima Región de Los Lagos. Tesis de Licenciatura, Escuela de Medicina Veterinaria, Universidad Austral de Chile, Valdivia, Chile.

Kiley-Worthington M. 1987. The behaviour of horses; in relation to management and training. JA Allen, London, UK.

Luescher UA, DB McKeown, J Halip. 1991. Reviewing the causes of obsessive-compulsive disorders in horses. Vet Med 86, 527-530.

Mac-Leod C. 1999. Estudio de los equinos carretoneros atendidos en un policlínico en Valdivia, caracterizando aspectos de hipometría, patologías, alimentación, cascos y herrajes. Tesis de Licenciatura, Escuela de Medicina Veterinaria, Universidad Austral de Chile, Valdivia, Chile.
Márquez C. 2009. Elaboración y aplicación de una pauta de bienestar animal en equinos estabulados. Memoria de título, Escuela de Medicina Veterinaria, Universidad Austral de Chile, Valdivia, Chile.

McGreevy PD, PJ Cripps, NP French, LE Green, CJ Nicol. 1995. Management factors associated with stereotypic and redirected behaviour in the Thoroughbred horse. Equine Vet J 27, 86-91.

McGreevy P. 2004. Equine behavior: A guide for veterinarians and equine scientists. Saunders, London, England.

Mills DS, KD Taylor, JJ Cooper. 2005. Weaving, headshaking, cribbing, and other stereotypies. American Association of Equine Practitioners. Lexington, KY, USA.

Morán G, O Araya, H Folch. 2006. Obstrucción recurrente de las vías aéreas en el caballo. Arch Med Vet 38, 201-217.

Muñoz R. 2004. Estudio descriptivo y comparativo de dos sistemas de amansa en caballos criollos chilenos. Memoria de título, Escuela de Medicina Veterinaria, Universidad Austral de Chile, Valdivia, Chile.

Muñoz L, J Torres, O Sepúlveda, C Rehhof, R Ortiz. 2009. Frecuencia de comportamientos anormales estereotipados en caballos chilenos. Arch Med Vet 41, 73-76.

Naour E. 2003. Elaboración de una guía de consejos prácticos para el manejo de los caballos carretoneros de Valdivia. Memoria de título, Escuela de Medicina Veterinaria, Universidad Austral de Chile, Valdivia, Chile.

Ninomiya S, R Kusunose, S Sato, M Terada, K Sugawara. 2004. Effects of feeding methods on eating frustration in horses. Anim Sci J 75, 465-469.

Pell SM, PD McGreevy. 1999. Prevalence of stereotypic and other problem behaviours in Thoroughbred horses. Aust Vet J 77, 678-679.

Porte E. 1979. Cría, doma y arreglo del caballo a la chilena. Editorial Universitaria SA, Santiago, Chile.

Pritchard JC, AC Lindberg, DC Main, HR Whay. 2005. Assessment of the welfare of working horses, mules and donkeys, using health and behaviour parameters. Prev Vet Med 69, 265-283.

Retamales NR. 1989. Reproducción, criaza y manejo de un haras F.S. de carrera. Ediciones Mar del Plata, Santiago, Chile.

Tadich T, A Escobar, RA Pearson. 2008. Husbandry and welfare aspects of urban draught horses in the south of Chile. Arch Med Vet 40, 267-273.

Tadich T, O Araya. 2010. Conductas no deseadas en equinos. Arch Med Vet 42, 29-41.

Vecchiotti G, R Galanti. 1986. Evidence of heredity of crib-biting, weaving and stall-walking in Thoroughbred horses. Livest Prod Sci 14, 91-95.

von Witzendorff C, I Quintana, G Sievers, T Schnieder, G SamsonHimmelstjerna. 2003. Estudio sobre resistencia frente a los bencimidazoles de pequeños estróngilos (Cyathostominae) del equino en el sur de Chile. Arch Med Vet 35, 187-194.

Waters AJ, CJ Nicol, NP French. 2002. Factors influencing the development of stereotypic and redirected behaviours in young horses: findings of a four year prospective epidemiological study. Equine Vet J 34, 572-579.

Webster A, A Clarke, T Madelin, C Wathes. 1987. Air hygiene in stables 1: effects of stable design, ventilation and management on the concentration of respirable dust. Equine Vet J 19, 448-453.

Wood J, J Holder, D Main. 1998. Quality assurance schemes. Meat Sci 49, Suppl. 1: S191-1203.

WSPA, World Society for the Protection of Animal. 2003. Cuidados básicos para equinos. Kalion SA, Bogotá, Colombia. 